\title{
A CLASS OF UNIVALENT FUNCTIONS WITH VARYING ARGUMENTS
}

\author{
K. S. PADMANABHAN and M. JaYAMALA \\ The Ramanujan Institute, \\ University of Madras, \\ Madras - 600005 , \\ and \\ Department of Mathematics, \\ Queen Mary's College, \\ Madras - 600005 , \\ India.
}

(Received April 24, 1990 and in revised form August 7, 1991)

ABSTRACT. $f(z)=z+\sum_{m=2}^{\infty} a_{m} z^{m}$ is said to be in $v\left(\theta_{n}\right)$ if the analytic and univalent function $f$ in the unit disc $E$ is normalised by $f(0)=0, f^{\prime}(0)=1$ and arg $a_{n}=\theta_{n}$ for all $n$. If further there exists a real number $\beta$ such that $\theta_{n}+(n-1) \beta \equiv \pi(\bmod 2 \pi)$ then $f$ is said to be in $v\left(\theta_{n}, \beta\right)$. The union of $v\left(\theta_{n}, \beta\right)$ taken over all possible sequence $\left\{\theta_{n}\right\}$ and all possible real number $\beta$ is denoted by $V$. $v_{n}(A, B)$ consists of functions $f \varepsilon V$ such that

$$
\frac{D^{n+1} f(z)}{D^{n} f(z)}=\frac{1+A w(z)}{1+B w(z)} \text {, }
$$

$-1 \leq A<B \leq 1$, where $n \varepsilon N \cup\{0\}$ and $w(z)$ is analytic, $w(0)=0$ and $|w(z)|<1, z \varepsilon E$. In this paper we find the coefficient inequalities, and prove distortion theorems.

KEY WORDS AND PHRASES. Varying arguments, Ruscheweyh derivative, Distortion theorems, Coefficient estimates. MATHEMATICS SUBJECT CLASSIFICATION No. $30 \mathrm{CA5}$

\section{INTRODUCTION.}

Let $A$ denote the class of functions $f(z)$ analytic in the unit disc $E=\{z:|z|<1\}$. Let $S$ denote the subclass of $A$ consisting functions normalised by $f(0)=0$ and $f^{\prime}(0)=1$ which are univalent in $E$. The Hadamard product $\left(f^{*} g\right)(z)$ of two functions

$$
\begin{aligned}
f(z)=\sum_{m=0}^{\infty} a_{m} z^{m} \text { and } g(z) & =\sum_{m=0}^{\infty} b_{m} z^{m} \text { in A is given by, } \\
\left(f^{*} g\right)(z) & =\sum_{m=0}^{\infty} a_{m} b_{m} z^{m} .
\end{aligned}
$$


Let $D^{n} f(z)=\frac{z}{(1-z)^{n+1}} * f(z), n \in N \cup\{0\}$ where $N=\{1,2,3, \ldots\}$. Ruscheweyh [2] observed that $D^{n} f(z)=z\left(z^{n-1} f(z)\right)^{(n) / n !} \cdot D^{n} f(z)$ is called the $n^{\text {th }}$ Ruscheweyh derivative of $f(z)$ by $A l-A m i r i$ [1].

DEFINITION 1. (Silverman [3]). $f(z)=z+\sum_{m=2}^{\infty} a_{m} z^{m}$ is said to be in $v\left(\theta_{n}\right)$ if $f \varepsilon s$ and arg $a_{n}=\theta_{n}$ for all $n$. If further there exists a real number $\beta$ such that $\theta_{n}+(n-1) \beta \equiv \pi(\bmod 2 \pi)$, then $f$ is said to be in $v\left(\theta_{n}, \beta\right)$. The union of $v\left(\theta_{n}, \beta\right)$ taken overall possible sequences $\left\{\theta_{n}\right\}$ and all possible real number $f$ is denoted by $V$.

Now we define the class $V_{n}(A, B)$ consisting of functions $f \varepsilon V$ such that $\frac{D^{n+1} f(z)}{D^{n} f(z)}=\frac{1+A w(z)}{1+B w(z)},-1 \leq A<B \leq 1$, where $n \in N \cup\{0\}$ and $w(z)$ is analytic, $w(0)=0$ and $|w(z)|<1$, $z \varepsilon E$. Let $K_{n}(A, B)$ denote the class of functions $f \varepsilon V$ such that $z f^{\prime}(z) \varepsilon V_{n}(A, B)$.

2. COEFFICIENT INEQUALITIES.

THEOREM 1. Let $f \varepsilon V$. Then $f \varepsilon V_{n}(A, B)$ if and only if

$$
\sum_{m=2}^{\infty} \frac{(n+m-1) !}{(n+1) !(m-1) !} C_{m}\left|a_{m}\right|<(B-A) \text {. }
$$

where $C_{m}=(B+1)(n+m)-(1+A)(n+1)$.

PROOF. Suppose $f \varepsilon V_{n}(A, B)$. Then

$$
\frac{D^{n+1} f(z)}{D^{n} f(z)}=\frac{1+A w(z)}{1+B w(z)},-1 \leq A<B \leq 1
$$

$w(z)$ is analytic, $w(0)=0$ and $|w(z)|<1, z \varepsilon E$. We get

$$
w(z)=\frac{D^{n} f(z)-D^{n+1} f(z)}{B D^{n+1} f(z)-A D^{n} f(z)} \text {. }
$$

Since Re $w(z)<|w(z)|<1$, we obtain on simplification,

$\operatorname{Re}\left\{\frac{\sum_{m=2}^{\infty} \frac{(n+m-1) !}{(n+1) !(m-1) !}[(n+1)-(n+m)] a_{m} z^{m-1}}{(B-A)+\sum_{m=2}^{\infty} \frac{(n+m-1) !}{(n+1) !(m-1) !}[B(n+m)-A(n+1)] a_{m} 2^{m-1}}\right\}<1$.

Since $f \varepsilon V, f$ lies in $v\left(\theta_{m}, \beta\right)$ for some sequence $\left\{\theta_{m}\right\}$ and a real number $\beta$ such that

$$
\theta_{m}+(m-1) \beta \equiv x(\bmod 2 \pi) \text {. Set } z=r e^{1 \beta} \text {. }
$$


Then we get,

$$
\begin{aligned}
& \therefore=\left\{\frac{\sum_{m=2}^{\infty} \frac{(n+m-1) !}{(n+1) !(m-1) !}[(n+1)-(n+m)]\left|a_{m}\right| r^{m-1} e^{i\left(\theta_{m}+\overline{m-1} \beta\right)}}{\left.(B-A)+\sum_{m=2}^{\infty} \frac{(n+m-1) !}{(n+1) !(m-1) !}[B(n+m)-A(n+1)]\left|a_{m}\right| r^{m-1} e^{i\left(\theta_{m}+m-1\right.} B\right)}\right\} \\
& \quad<1 . \\
& \sum_{m=2}^{\infty} \frac{(n+m-1) !}{(n+1) !(m-1) !}[(n+m)-(n+1)]\left|a_{m}\right| r^{m-1} \\
& \quad<(B-A)-\sum_{m=2}^{\infty}-\frac{(n+m-1) !}{(n+1) !(m-1) !}[B(n+m)-A(n+1)]\left|a_{m}\right| r^{m-1} \\
& \sum_{m=2}^{\infty} \frac{(n+m-1) !}{(n+1) !(m-1) !}[(B+1)(n+m)-(1-A)(n+1)]\left|a_{m}\right| r^{m-1}<(B-A)
\end{aligned}
$$

Hence,

$$
\sum_{m=2}^{\infty} \frac{(n+m-1) !}{(n+1) !(m-1) !} c_{m}\left|a_{m}\right| r^{m-1}<(B-A) .
$$

Letting $r \rightarrow 1$ we get $(2.1)$.

Conversely, suppose $f \varepsilon V$ and satisfies (2.1). In view of (2.4) which is implied by $(2.1)$, since $r^{m-1}<1$, we have,

$$
\begin{aligned}
\left.\right|_{m=2} ^{\infty} \frac{(n+m-1) !}{(n+1) !(m-1) !}[(n+1)-(n+m)] a_{m} z^{m-1} \mid \\
\quad \leq \sum_{m=2}^{\infty} \frac{(n+m-1) !}{(n+1) !(m-1) !}[(n+m)-(n+1)]\left|a_{m}\right| r^{m-1} \\
\quad<(B-A)-\sum_{m=2}^{\infty} \frac{(n+m-1) !}{(n+1) !(m-1) !}[B(n+m)-A(n+1)]\left|a_{m}\right| r^{m-1} \\
\quad \leq\left|(B-A)-\sum_{m=2}^{\infty} \frac{(n+m-1) !}{(n+1) !(m-1) !}[A(n+1)-B(n+m)] a_{m} z^{m-1}\right|
\end{aligned}
$$

which gives $(2.2)$ and hence follows that $f \varepsilon v_{n}(A, B)$.

COROLLARY 1. If $f \varepsilon v$ is in $V_{n}(A, B)$ then,

$$
\left|a_{m}\right| \leq \frac{(n+1):(m-1):(B-A)}{(n+m-1) ! C_{m}}
$$

for $m \geq 2$. The equality holds for the function $f$ given by,

$$
f(z)=z+\frac{(n+1) !(m-1) !(B-A)}{(n+m-1) ! C_{m}} e^{1 \theta_{m}} z^{m}, z \varepsilon E .
$$

THEOAEM 2. Let $f \varepsilon v$. Then $f(z)=z+\sum_{m=2}^{\infty} a_{m} z^{m}$ is in $K_{n}(A, B)$ if and only if

$$
\sum_{m=2}^{\infty} \frac{(n+m-1) !}{(n+1) !(m-1) !} m c_{m}\left|a_{m}\right|<(B-A) .
$$


THEOREM 3. Let $f(z)=z+\sum_{m=2}^{\infty} a_{m} z^{m} \varepsilon v_{n}(A, B)$, with arg $a_{m}=\theta_{m}$ where $\left[\theta_{m}+(m-1) E\right] \equiv \pi(\bmod 2 \pi)$. Define $f_{1}(z)=z$ and $f_{m}(z)=z+\frac{(n+1) !(m-1) !(B-A) e^{1 \theta_{m} m}}{(n+m-1) ! C m}, m=2,3, \ldots, z \varepsilon E$. $f \varepsilon V_{n}(A, B)$ if and only if $f$ can be expressed as $f(z)=\sum_{m=1}^{\infty} \mu_{m} f_{m}(z)$ where $\mu_{m} \geq 0$ and $\sum_{m=1}^{\infty} \mu_{m}=1$.

PROOF. If $f(z)=\sum_{m=1}^{\infty} \mu_{m} f_{m}(z)$ with $\sum_{m=1}^{\infty} \mu_{m}=1, \mu_{m} \geq 0$, then,

$$
\begin{gathered}
\sum_{m=2}^{\infty} \frac{(n+m-1) ! C_{m} \mu_{m}}{(n+1) !(m-1) !} \cdot \frac{(n+1):(m-1) !(B-A)}{(n+m-1) ! C_{m}} \\
=\sum_{m=2}^{\infty} \mu_{m}(B-A)=\left(1-\mu_{1}\right)(B-A) \leq(B-A) .
\end{gathered}
$$

Hence $f \varepsilon v_{n}(A, B)$.

Conversely, let

$$
\begin{gathered}
f(z)=2+\sum_{m=2}^{\infty} a_{m} z^{m} \varepsilon v_{n}(A, B), \\
\text { define, } \mu_{m}=\frac{(n+m-1):\left|a_{m}\right| C_{m}}{(n+1) !(m-1) !(B-A)}, m=2,3, \ldots \text { and define }
\end{gathered}
$$
$\mu_{1}=1-\sum_{m=2}^{\infty} \mu_{m}$. From Theorem $1, \sum_{m=2}^{\infty} \mu_{m} \leq 1$ and $80 \mu_{1} \geq 0$. Since, $\mu_{m} f_{m}(z)=\mu_{m} z+a_{m} z^{m}$.

$$
\sum_{m=1}^{\infty} \mu_{m} f_{m}(z)=z+\sum_{m=2}^{\infty} a_{m} z^{m}=f(z) \text {. }
$$

THEOREM 4. Define $f_{1}(z)=2$ and

$$
f_{m}(z)=z+\frac{e^{1 \theta_{m}}(n+1) !(m-1) !(B-A) z^{m}}{(n+m-1) ! m C_{m}}, m=2,3, \ldots z \varepsilon E .
$$

Then $f \varepsilon K_{n}(A, B)$ if and only if $f$ can be expressed as

$f(z)=\sum_{m=1}^{\infty} \mu_{m} f_{m}(z)$ where $\mu_{m} \geq 0$ and $\sum_{m=1}^{\infty} \mu_{m}=1$.

3. DISTORTION THEOREMS.

THEOREM 5. Let the function $f(z)=z+\sum_{m=2}^{\infty} a_{m} z^{m}$ be in the class $V_{n}(A, B)$. Then,

$$
\begin{aligned}
& |z|-(B-A)|z|^{2} / C_{2} \leq|f(z)| \leq|z|+(B-A)|z|^{2} / C_{2} \\
& 1-2(B-A)|z| / C_{2} \leq\left|f^{\prime}(z)\right| \leq 1+2(B-A)|z| / C_{2} \cdot \\
& \text { PROOF. }|f(z)|=\left|z+\sum_{m=2}^{\infty} a_{m} z^{m}\right| \leq|z|+|z|^{2} \sum_{m=2}^{\infty}\left|a_{m}\right|
\end{aligned}
$$


and $|f(z)| \geq|z|-\left.|z|\right|_{m=2} ^{\infty}\left|a_{m}\right|$. Since $\frac{(n+m-1) ! C_{m}}{(n+1) !(m-1) !}$ is an increasing function of $m \geq 2$ and $f(z) \varepsilon V_{n}(A, B)$, by Theorem 1 , we have

$$
\frac{(n+1):}{(n+1) ! 1 !} c_{2} \sum_{m=2}^{\infty}\left|a_{m}\right| \leq \sum_{m=2}^{\infty} \frac{(n+m-1) !}{(n+1) !(m-1) !} c_{m}\left|a_{m}\right| \leq(B-A)
$$

that is,

$$
\sum_{m=2}^{\infty}\left|a_{m}\right| \leq \frac{B-A}{C_{2}}
$$

From (3.3) we get (3.1)

$$
\left|f^{\prime}(z)\right|=\left|1+\sum_{m=2}^{\infty} m a_{m} z^{m-1}\right| \leq 1+|z| \sum_{m=2}^{\infty} m\left|a_{m}\right|
$$

and

$$
|f \cdot(z)| \geq 1-|z| \sum_{m=2}^{\infty} m\left|a_{m}\right| \text {. }
$$

Since $\frac{(n+m-1) ! C_{m}}{(n+1) ! m !}$ is an increasing function of $m \geq 2$ and $\frac{(n+m-1) ! m C_{m}}{(n+1) !(m+1) !}<\frac{(n+m-1) ! m C_{m}}{(n+1) ! m !}$ by Theorem 1, we have,

$$
\frac{(n+1) ! C_{2}}{(n+1) ! 2} \sum_{m=2}^{\infty} m\left|a_{m}\right| \leq \sum_{m=2}^{\infty} \frac{(n+m-1) ! c_{m}\left|a_{m}\right|}{(n+1) !(m-1) !} \leq(B-A)
$$

that is,

$$
\sum_{m=2}^{\infty} m\left|a_{m}\right| \leq \frac{2(B-A)}{C_{2}}
$$

From (3.4) we get (3.2). Further for the function $f(z)=z_{2}+\frac{(B-A)}{C_{2}} z^{2}$, we can see that the results of the Theorem are sharp.

COROLLARY 2. Let $f(z)=z+\sum_{m=2}^{\infty} a_{m} z^{m}$ be in the class $v_{n}(A, B)$. Then $f(z)$ is included in a disc with its center at the origin and radius $I$ given by $I=\left(C_{2}+B-A\right) / C_{2}$ and $f^{\prime}(z)$ is included in a disc with its center at the origin and radius $r_{1}$ given by $r_{1}=\left[C_{2}+2(B-A)\right] / C_{2}$.

THEOREM 6. Let the function $f(z)=z+\sum_{m=2}^{\infty} a_{m} z^{m}$ be in the class $K_{n}(A, B)$, then,

$$
|z|-(B-A)|z|^{2} / 2 C_{2} \leq|f(z)| \leq|z|+(B-A)|z|^{2} / 2 C_{2}
$$

and

$$
1-(B-A)|z| / C_{2} \leq\left|f^{\prime}(z)\right| \leq 1+(B-A)|z| / C_{2}
$$

for $z \varepsilon E$. The results are sharp for the function $f(z)=z+(B-A) z^{2} / 2 C_{2}$. 


\section{REFERENCES}

1. H.S.Al-Amiri, On Ruscheweyh derivatives, Ann. Polon, Math. 38 $(1980), 87-94$.

2. S.Ruscheweyh, New criteria for univalent functions, Proc. Am. Math. Soc. 49 (1975), 109-115.

3. H.Silverman, Univalent Functions with varying Arguments, Houston Journal of Math. Vol.7, No.2 (1981). 


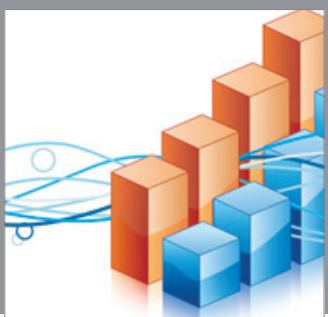

Advances in

Operations Research

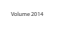

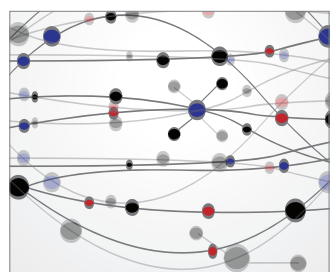

\section{The Scientific} World Journal
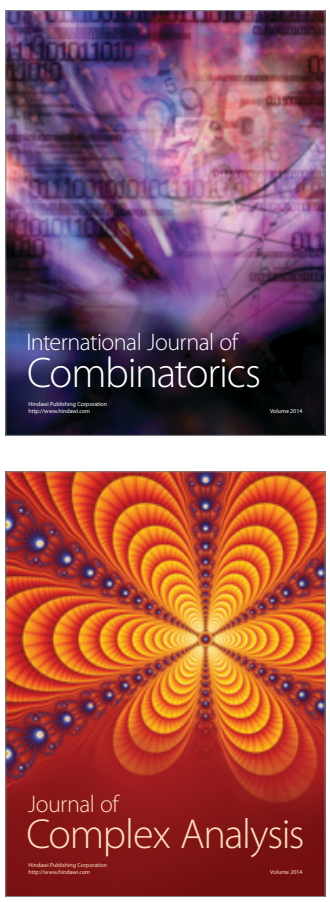

International Journal of

Mathematics and

Mathematical

Sciences
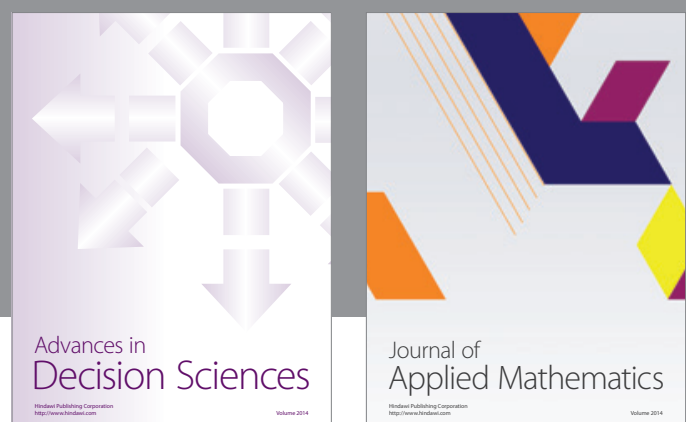

Journal of

Applied Mathematics
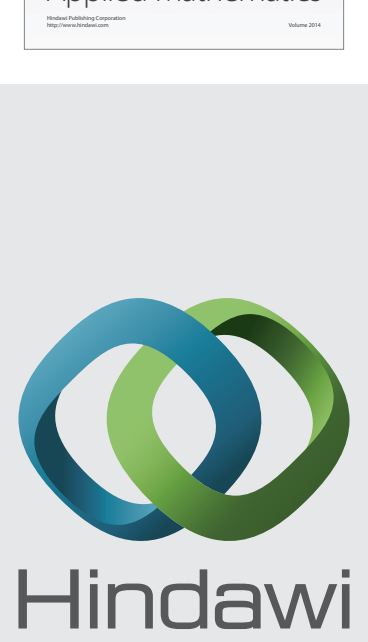

Submit your manuscripts at http://www.hindawi.com
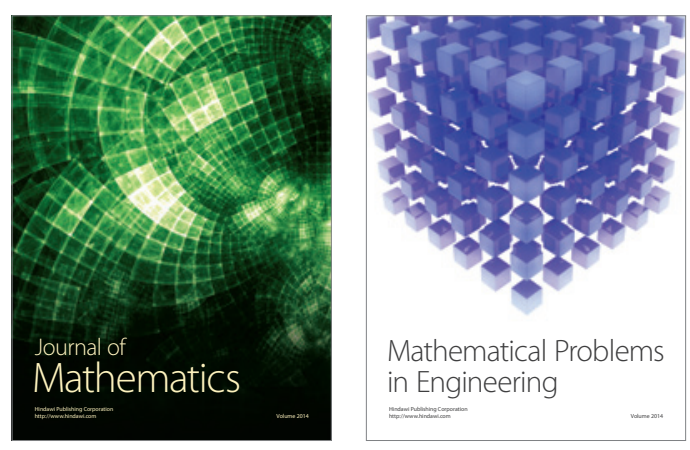

Mathematical Problems in Engineering
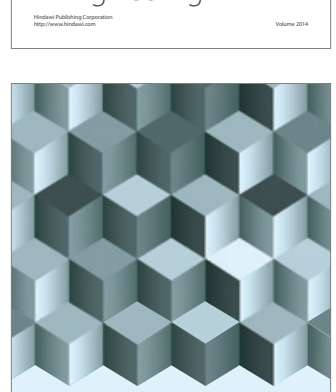

Journal of

Function Spaces
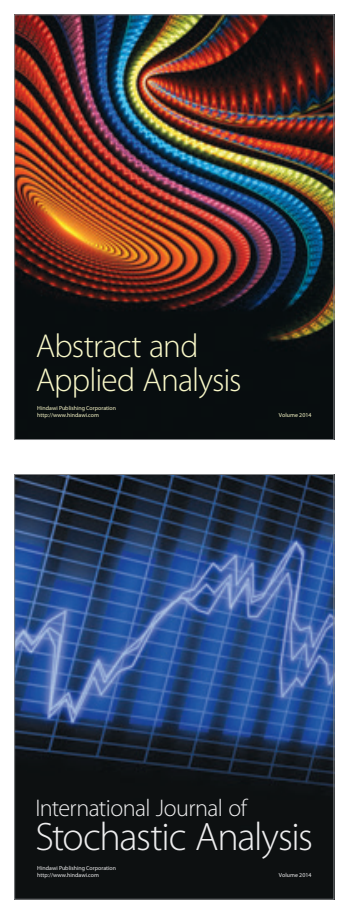

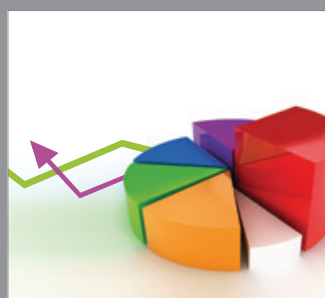

ournal of

Probability and Statistics

Promensencen
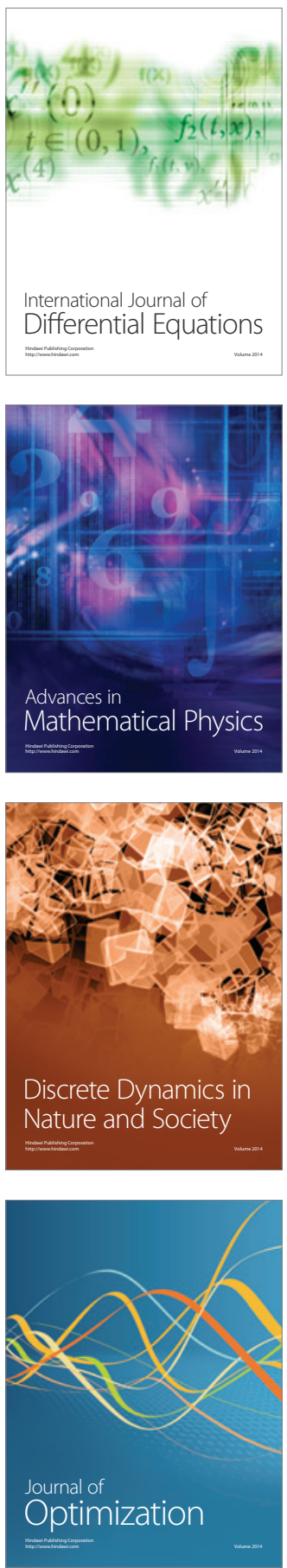